\title{
Two Groups in a Curie-Weiss Model with Heterogeneous Coupling
}

\author{
Werner Kirsch and Gabor Toth
}

Fakultät für Mathematik und Informatik

FernUniversität Hagen

D-58084 Hagen

Germany

email: werner.kirsch@fernuni-hagen.deｇabor.toth@fernuni-hagen.de

\begin{abstract}
We discuss a Curie-Weiss model with two groups with different coupling constants within and between groups. For the total magnetisations in each group, we show bivariate laws of large numbers and a central limit theorem which is valid in the high temperature regime. In the critical regime, the total magnetisation normalised by $N^{3 / 4}$ converges to a non-trivial distribution which is not Gaussian, just as in the single-group Curie-Weiss model. Finally, we prove a kind of a 'law of large numbers' in the low temperature regime, more precisely we prove that the empirical magnetisation converges in distribution to a mixture of two Dirac measures.

Keywords: Curie-Weiss; Central Limit Theorems; Multi-Population Models

2010 Mathematics Subject Classification: 60F05; 82B20
\end{abstract}

\section{Introduction}

The Curie-Weiss model is probably the easiest model of magnetism which shows a phase transition between a paramagnetic and a ferromagnetic phase. In this model the spins can take values in $\{-1,1\}$ (or up/down), each spin interacts with all the others in the same way. More precisely, for finitely many spins $X:=\left(X_{1}, X_{2}, \ldots, X_{N}\right) \in\{-1,1\}$ the energy of the spins is given by

$$
H=H\left(X_{1}, \ldots, X_{N}\right):=-\frac{1}{2 N}\left(\sum_{j=1}^{N} X_{j}\right)^{2} .
$$


The 'Gibbs measure' or 'canonical ensemble' with coupling constant $(\approx$ inverse temperature) $J_{0} \geq 0$ the probability of a spin configuration is given by

$$
\mathbb{P}\left(X_{1}=x_{1}, \ldots, X_{N}=x_{N}\right):=Z^{-1} e^{-J_{0} H\left(x_{1}, \ldots, x_{N}\right)}
$$

where $x_{i} \in\{-1,1\}$ and $Z$ is a normalisation constant which depends on $N$ and $J_{0}$.

The quantity

$$
S_{N}=\sum_{j=1}^{N} X_{j}
$$

is called the (total) magnetisation. It is well known (see e.g. Ellis [1] or [14] that the Curie-Weiss model has a phase transition at $J_{0}=1$ in the following sense

$$
\frac{1}{N} S_{N} \Longrightarrow \frac{1}{2}\left(\delta_{-m\left(J_{0}\right)}+\delta_{m\left(J_{0}\right)}\right)
$$

where $\Rightarrow$ denotes convergence in distribution, $\delta_{x}$ the Dirac measure in $x$.

For $J_{0} \leq 1$ we have $m\left(J_{0}\right)=0$ which is the unique solution of

$$
\tanh \left(J_{0} x\right)=x
$$

eq:mJ_0

for this case.

If $J_{0}>1$ equation (leg: positive one.

Equation (䢗) $1 \mathrm{ln}$ a substitute for the law of large numbers for i.i.d. random variables.

Moreover, for $J_{0}<1$ there is a central limit theorem, i. e.

$$
\frac{1}{\sqrt{N}} S_{N} \Longrightarrow \mathcal{N}\left(0, \frac{1}{1-J_{0}}\right)
$$

There is a huge amount of literature on the Curie-Weiss model. We can just mention a few papers here. The Curie-Weiss model is also called the HusimiTemperley model. It was first introduced by Husimi li1 and Temperley [ZU]. Subsequently, it was discussed by Kac [13 and Ellis and Newman 66, 7. It was also treated in the textbooks Thompson $\frac{\text { hompson }}{21}$, and Ellis $[5$.

More recently, the Curie-Weiss model has been used in the context of social and political interactions. See e.g. [4, $\frac{10}{10}$, .

In this paper we consider two groups of Curie-Weiss spins $X=\left(X_{1}, \ldots, X_{N_{1}}\right)$ and $Y=\left(Y_{1}, \ldots, Y_{N_{2}}\right)$ with $N:=N_{1}+N_{2}$. The spins $X$ and $Y$ are CurieWeiss spins with coupling constant $J_{1}$ and $J_{2}$ respectively, in addition there is a Curie-Weiss-type interaction between the $X_{i}$ and the $Y_{j}$ with coupling constant $\bar{J}$. 
We set

$$
J:=\left(\begin{array}{cc}
J_{1} & \bar{J} \\
\bar{J} & J_{2}
\end{array}\right)
$$

and assume that $J_{1}, J_{2}, \bar{J}>0$ and

$$
\Delta:=J_{1} J_{2}-\bar{J}^{2}>0,
$$

eq:delta

so that the matrix $J$ is positive definite. Loosely speaking, this conditions ensures that the interaction within groups dominates the interaction between the groups.

The energy function is given by

$$
H=H_{J}(X, Y):=-\frac{1}{2 N}\left[J_{1}\left(\sum_{j=1}^{N_{1}} X_{j}\right)^{2}+J_{2}\left(\sum_{j=1}^{N_{2}} Y_{j}\right)^{2}+2 \bar{J} \sum_{i=1}^{N_{1}} \sum_{j=1}^{N_{2}} X_{i} Y_{j}\right] .
$$

We denote the Gibbs measure associated with $H_{J}(X, Y)$ by $\mathbb{P}_{J}$ (sometimes abbreviated by $\mathbb{P}$ ) defined by

$$
\mathbb{P}_{J}(A):=Z^{-1} \sum_{(X, Y) \in A} e^{-H_{J}(X, Y)}
$$

where $Z$ is a normalizing constant which makes $\mathbb{P}_{J}$ a probability measure. The corresponding expectation is called $\mathbb{E}_{J}$, sometimes abbreviated $\mathbb{E}$.

By sending $N$ to infinity we mean that both $N_{1}$ and $N_{2}$ tend to infinity. We set

$$
\alpha_{1}:=\lim \frac{N_{1}}{N}, \quad \alpha_{2}:=\lim _{N \rightarrow \infty} \frac{N_{2}}{N}=1-\alpha_{1}
$$

and assume that these limits exist and $0<\alpha_{1}<1$.

In this paper we consider the asymptotic behaviour of the two-dimensional random variables

$$
\left(\frac{1}{N_{1}^{\gamma}} \sum_{i=1}^{N_{1}} X_{i}, \frac{1}{N_{2}^{\gamma}} \sum_{j=1}^{N_{2}} Y_{j}\right)
$$

where $\gamma=1, \frac{1}{2}$ or $\frac{3}{4}$ depending on the parameters of the model, namely $J_{1}, J_{2}, \bar{J}$ and $\alpha_{1}, \alpha_{2}$.

Our assumptions on $J_{1}, J_{2}$ and $\bar{J}$ exclude a few 'borderline' cases. If $\bar{J}=0$ the two groups are independent of each other and can therefore be treated as independent single group Curie-Weiss spins. This is also the case if $J_{1}=0$ or $J_{2}=0$ as this implies $\bar{J}=0$ by assumption (B).

Condition 迎: delta which we callthe homogeneous one requires a somewhat different technique. It is treated in $\frac{16}{16}$. 
Another borderline case is given by $\alpha_{1}=0$ or $\alpha_{2}=0$ (assuming still that both $N_{1}$ and $N_{2}$ tend to infinity). We may even consider the following extension of our model: The groups may consist of $\tilde{N}_{i} \approx \rho_{i} N$ but the averages in (II2) are taken over $N_{i} \approx \alpha_{i} N \leq \tilde{N}_{i}$.

These cases can be treated by the techniques of this paper as well. With the obvious changes, the results and their proofs remain valid for these extensions. In order to avoid a notational overkill we stick to the stronger assumptions made.

\subsection{High Temperature Regime}

The parameter space of this model is

$$
\Phi:=\left\{\left(J_{1}, J_{2}, \bar{J}, \alpha_{1}, \alpha_{2}\right) \in(0, \infty)^{5} \mid J_{1} J_{2}>\bar{J}^{2}, \alpha_{1}+\alpha_{2}=1\right\} .
$$

For the single-group model, the high temperature regime is quite simply expressed by the single condition $J_{0}<1$. For two groups with a heterogeneous coupling matrix, we have a very different situation: each within-group coupling constant $J_{\nu}$ has to be small in relation to the reciprocal of the group's size. Once the within-group couplings have been chosen, the between-groups coupling has to be small, too. How small depends on how close the other two couplings are to the reciprocals of the group sizes. If the within-group couplings are very small, that leaves more leeway for the between-groups coupling to be larger.

We shall assume that the interactions satisfy

$$
\begin{aligned}
J_{1} & <\frac{1}{\alpha_{1}} \\
J_{2} & <\frac{1}{\alpha_{2}} \\
\bar{J}^{2} & <\left(\frac{1}{\alpha_{1}}-J_{1}\right)\left(\frac{1}{\alpha_{2}}-J_{2}\right) .
\end{aligned}
$$

and refer to these conditions as the 'high temperature regime', and we shall also refer to the subset $\Phi_{h}$ of $\Phi$ where these conditions hold by the same name. Note that if we use the symbol $\alpha$ for the diagonal $2 \times 2$ matrix with entries $\alpha_{1}$ and $\alpha_{2}$, we can formulate these conditions equivalently in matrix form: the matrix

$$
J^{-1}-\alpha
$$

is positive definite if and only if we are in the high temperature regime (see Proposition (II).

We prove a 'law of large numbers'.

LLN Theorem 1. In the high temperature regime, we have 


$$
\left(\frac{1}{N_{1}} \sum_{i=1}^{N_{1}} X_{i}, \frac{1}{N_{2}} \sum_{j=1}^{N_{2}} Y_{j}\right) \underset{N \rightarrow \infty}{\Longrightarrow}(0,0) .
$$

Above ' $\Longrightarrow$ ' denotes convergence in distribution of the 2-dimensional random variable on the left hand side.

We also have a 'central limit theorem'. Using $\alpha=\left(\begin{array}{cc}\alpha_{1} & 0 \\ 0 & \alpha_{2}\end{array}\right)$ we define the matrix

$$
C=1+\left(\alpha^{-1 / 2} J^{-1} \alpha^{-1 / 2}-1\right)^{-1}
$$

eq: covariance

where 1 denotes the identity matrix.

CLT Theorem 2. In the high temperature regime, we have

$$
\left(\frac{1}{\sqrt{N_{1}}} \sum_{i=1}^{N_{1}} X_{i}, \frac{1}{\sqrt{N_{2}}} \sum_{j=1}^{N_{2}} Y_{j}\right) \underset{N \rightarrow \infty}{\Longrightarrow} \mathcal{N}((0,0), C),
$$

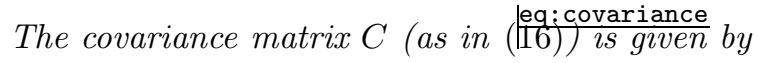

$$
C=\frac{1}{\left(1-\alpha_{1} J_{1}\right)\left(1-\alpha_{2} J_{2}\right)-\alpha_{1} \alpha_{2} \bar{J}^{2}}\left[\begin{array}{cc}
1-\alpha_{2} J_{2} & \sqrt{\alpha_{1} \alpha_{2}} \bar{J} \\
\sqrt{\alpha_{1} \alpha_{2}} \bar{J} & 1-\alpha_{1} J_{1}
\end{array}\right]
$$

Remark 3. Theorem[implies that also expressions like $\frac{1}{\sqrt{N_{1}}} \sum_{i=1}^{N_{1}} X_{i} \pm \frac{1}{\sqrt{N_{2}}} \sum_{j=1}^{N_{2}} Y_{j}$ are asymptotically Gaussian distributed.

\subsection{Critical Regime}

The critical regime is where $\left(\sum_{i=1}^{N_{1}} X_{i}, \sum_{j=1}^{N_{2}} Y_{j}\right)$ abruptly changes behaviour. In the single-group model, this occurs at $J_{0}=1$. For two groups with a heterogeneous coupling matrix, in the critical regime, each within-group coupling constant $J_{\nu}$ has to be small in relation to the reciprocal of the group's size. Once the within-group couplings have been chosen, the between-groups coupling has to have an exact magnitude, which is larger than in the high temperature regime:

$$
\begin{aligned}
J_{1} & <\frac{1}{\alpha_{1}} \\
J_{2} & <\frac{1}{\alpha_{2}} \\
\bar{J}^{2} & =\left(\frac{1}{\alpha_{1}}-J_{1}\right)\left(\frac{1}{\alpha_{2}}-J_{2}\right) .
\end{aligned}
$$

We shall call the subset of $\Phi$ where these conditions hold $\Phi_{c}$ and we can also formulate these conditions equivalently in matrix form: the matrix 


$$
J^{-1}-\alpha
$$

is singular and has positive diagonal entries if and only if we are in the critical regime.

We also note that if $J_{1}=\frac{1}{\alpha_{1}}$ or $J_{2}=\frac{1}{\alpha_{2}}$ then $\frac{\text { legij } \text { condJ3-1-1 }}{(21) \text { mples }}=0$ hence the two groups are independent of each other and can be treated as in the single-group case.

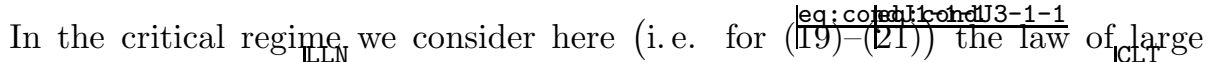

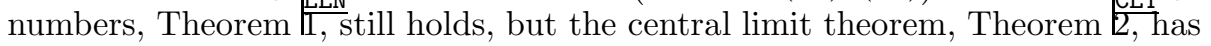
to be replaced by a theorem describing the asymptotic behaviour of

$$
T_{N}=\left(\frac{1}{N_{1}^{3 / 4}} S_{N_{1}}^{(1)}, \frac{1}{N_{2}^{3 / 4}} S_{N_{2}}^{(2)}\right) .
$$

This sequence $T_{N}$ converges in distribution but not to a normal distribution. We state the moments of the limiting measure in Theorem

The critical regime results are:

LLN-1 Theorem 4. In the critical regime, we have

$$
\left(\frac{1}{N_{1}} \sum_{i=1}^{N_{1}} X_{i}, \frac{1}{N_{2}} \sum_{j=1}^{N_{2}} Y_{j}\right) \underset{N \rightarrow \infty}{\Longrightarrow} \delta_{(0,0)} .
$$

If we choose as normalising factors $N_{\nu}^{\frac{3}{4}}$ instead of $N_{\nu}$, then we obtain

Fluctuations Theorem 5. In the critical regime, the random variables

$$
\left(\frac{1}{N_{1}^{3 / 4}} \sum_{i=1}^{N_{1}} X_{i}, \frac{1}{N_{2}^{3 / 4}} \sum_{j=1}^{N_{2}} Y_{j}\right)
$$

converge in distribution to a measure $\mu$ (on $\mathbb{R}^{2}$ ) with moments

$$
\begin{aligned}
m_{K, Q}:=\int x^{K} y^{Q} d \mu(x, y) \\
=\left[\frac{12}{\alpha_{1}\left(L_{2}-\alpha_{2}\right)^{2}+\alpha_{2}\left(L_{1}-\alpha_{1}\right)^{2}}\right]^{\frac{K+Q}{4}}\left(L_{1}-\alpha_{1}\right)^{\frac{Q}{2}}\left(L_{2}-\alpha_{2}\right)^{\frac{K}{2}} . \\
\quad \cdot \frac{\Gamma\left(\frac{K+Q+1}{4}\right)}{\Gamma\left(\frac{1}{4}\right)} \alpha_{1}^{\frac{K}{4}} \alpha_{2}^{\frac{Q}{4}} .
\end{aligned}
$$

where the matrix $L$ is defined by $L=\left(\begin{array}{cc}L_{1} & -\bar{L} \\ -\bar{L} & L_{2}\end{array}\right)=J^{-1}$ 


\subsection{Low Temperature Regime}

In the single-group model, the low temperature regime is characterised by the inequality $J_{0}>1$. The magnetization $\frac{1}{N} \sum X_{i}$ converges in distribution to the measure $\frac{1}{2}\left(\delta_{m^{*}}+\delta_{-m^{*}}\right)$ where $m^{*}$ is the unique positive solution of the equation $m=\tanh \left(J_{0} m\right)$. We regard this fact as a (substitute for the) law of large numbers.

In the case of two groups we define the low temperature regime to be the complement $\Phi_{l}:=\Phi \backslash\left(\Phi_{h} \cup \Phi_{c}\right)$ in the parameter space. We have a similar 'law of large numbers' in this case.

Theorem 6. In the low temperature regime, there are exactly two nonzero solutions $m^{*}=\left(m_{1}^{*}, m_{2}^{*}\right)$ and $-m^{*}$ of the system

$$
\begin{aligned}
m_{1} & =\tanh \left(J_{1} \alpha_{1} m_{1}-\bar{J} \alpha_{2} m_{2}\right) \\
\text { and } \quad & m_{2}=\tanh \left(J_{2} \alpha_{2} m_{2}-\bar{J} \alpha_{1} m_{1}\right) .
\end{aligned}
$$

We have

$$
\left(\frac{1}{N_{1}} \sum_{i=1}^{N_{1}} X_{i}, \frac{1}{N_{2}} \sum_{j=1}^{N_{2}} Y_{j}\right) \underset{N \rightarrow \infty}{\Longrightarrow} \frac{1}{2}\left(\delta_{-m^{*}}+\delta_{m^{*}}\right) .
$$

Moreover, we may assume $m_{1}^{*}>0$ and $m_{2}^{*}>0$.

Obviously, in the low temperature case, there can be no central limit theorem in the sense that $\left(\frac{1}{N_{1}^{\gamma}} \sum X_{i}, \frac{1}{N_{2}^{\gamma}} \sum Y_{i}\right)$ converges to a nontrivial limit measure. However, we conjecture that there is a 'conditional version' of a central limit theorem.

Acknowledgment: While finishing this paper we became aware of the papers [ 8 and which contain the above results as special cases. The methods used by those authors is very different from ours. We are grateful to Francesca Collet for drawing our attention to the papers $[\bar{z}$ and

We would also like to thank Matthias Löwe and Kristina Schubert $\frac{\pi S}{18}$ as well as an unnamed referee for valuable comments which in our opinion improved this paper considerably.

\section{A rough sketch of the proofs}

Our proofs are based on the method of moments, the basis of which is the following well known Theorem (see e. g. 位).

thm:moments Theorem 7. Suppose that $\mu_{N}$ and $\mu$ are probability measure on $\mathbb{R}^{d}$ for which all moments are finite and assume that $\mu$ is determined by its moments $m_{\underline{k}}(\mu)$. If $m_{\underline{k}}\left(\mu_{N}\right) \rightarrow m_{\underline{k}}(\mu)$ for all $\underline{k} \in \mathbb{N}^{d}$ as $N \rightarrow \infty$ then the measures $\mu_{N}$ converge weakly to $\mu$. 
It is also known that all (multidimensional) normal distributions are determined by their moments (see e. g. [17]).

Thus we will consider suitably normalized moments of the form

$$
\begin{aligned}
M_{K, Q} & :=\mathbb{E}_{J}\left(\left(\sum_{i=1}^{N_{1}} X_{i}\right)^{K}\left(\sum_{j=1}^{N_{2}} Y_{j}\right)^{Q}\right) \\
& =\sum_{i_{1}, i_{2}, \ldots, i_{K}} \sum_{j_{1}, j_{2}, \ldots, j_{Q}} \mathbb{E}_{J}\left(X_{i_{1}} \cdot \ldots \cdot X_{i_{K}} \cdot Y_{j_{1}} \cdot \ldots \cdot Y_{j_{Q}}\right) \\
& =\sum_{\underline{i} \in \mathcal{N}_{1}^{K}} \sum_{\underline{j} \in \mathcal{N}_{2}^{Q}} \mathbb{E}_{J}(X(\underline{i}) \cdot Y(\underline{j}))
\end{aligned}
$$

where $\underline{i}=\left(i_{1}, \ldots, i_{K}\right), \underline{j}=\left(j_{1}, \ldots, j_{Q}\right), \mathcal{N}_{1}=\left\{1,2, \ldots, N_{1}\right\}, \mathcal{N}_{2}=\left\{1,2, \ldots, N_{2}\right\}$ and $X(\underline{i})=\prod_{\nu=1}^{K} X_{i_{\nu}}$.

Since $X_{i}{ }^{2}=Y_{j}^{2}=1$ and due to exchangeability we have

$$
\begin{aligned}
& \mathbb{E}_{J}\left(X_{i_{1}} \cdot \ldots \cdot X_{i_{K}} \cdot Y_{j_{1}} \cdot \ldots \cdot Y_{j_{Q}}\right) \\
= & \mathbb{E}_{J}\left(X_{1} \cdot \ldots \cdot X_{\tilde{K}} \cdot Y_{1} \cdot \ldots \cdot Y_{\tilde{Q}}\right)
\end{aligned}
$$

eq: simpmoment

where $\tilde{K}$ (resp. $\tilde{Q})$ is the number of $i_{k}\left(\right.$ resp. $\left.j_{\ell}\right)$ which occur an odd number of times.

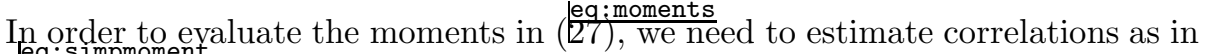
(28).

In section Sec: correl 13 we wiltprove asymptotic estimates for the correlations. For example in section 3.3 . Show that in the high temperature regime

$$
\left|\mathbb{E}_{J}\left(X_{1} \cdot \ldots \cdot X_{K} \cdot Y_{1} \cdot \ldots \cdot Y_{Q}\right)\right| \leq c_{K, Q} \frac{1}{N^{(K+Q) / 2}}
$$

eq: correl

where $c_{K, Q}$ depends on the matrix $J$ and the numbers $\alpha_{1}$ and $\alpha_{2}$ but not on $N$. Note that in the case of independent random variables, i. e. if $J=\left(\begin{array}{ll}0 & 0 \\ 0 & 0\end{array}\right)$ the correlation (Lej) correl

In a second step of the proof we need a 'bookkeeping' method, to keep track of the variety of terms in the sum ([DT). We have to count the number of simple, double, triple, etc. occurrences of the $X_{i}$ and $Y_{j}$ in (迸). We start the discussion of this topic with Lemma 22 A more precise discussion is given in subsection

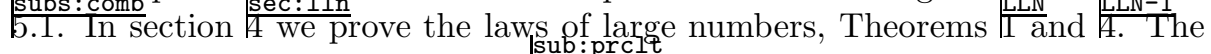
central limit theorem is proved in 5.3 combining the results on the correlations and the bookkeeping method. 


\section{Computing Expectations}

sec: correl

In this section we compute expectations (=correlations) of the form

$$
\mathbb{E}_{J}\left(X_{1} \cdot X_{2} \cdot \ldots \cdot X_{K} \cdot Y_{1} \cdot Y_{2} \cdot \ldots \cdot Y_{Q}\right)
$$

eq: correlations

asymptotically for the three regimes of $J$.

\subsection{A two-dimensional Hubbard-Stratonovich transform}

For any configuration of the spins

$$
(X, Y)=\left(X_{1}, X_{2}, \ldots, X_{N_{1}}, Y_{1}, Y_{2}, \ldots, Y_{N_{2}}\right)
$$

we set

$$
\begin{gathered}
S_{1}=\sum_{i=1}^{N_{1}} X_{i} \quad S_{2}=\sum_{j=1}^{N_{2}} Y_{j} \\
\text { and } \quad S=\left(\begin{array}{c}
S_{1} \\
S_{2}
\end{array}\right)
\end{gathered}
$$

and define the function

$$
\begin{aligned}
h\left(S_{1}, S_{2}\right) & =\frac{1}{2 N}\left(S_{1}, S_{2}\right) J\left(\begin{array}{c}
S_{1} \\
S_{2}
\end{array}\right) \\
& =\frac{1}{2 N} S^{\prime} J S,
\end{aligned}
$$

For a symmetric positive definite $2 \times 2$ matrix $A$ and a point $x_{0} \in \mathbb{R}^{2}$ we can use the following equality to express a value of the exponential function as an integral:

$$
e^{\frac{x_{0}^{\prime} A x_{0}}{2}}=\frac{\sqrt{\operatorname{det} A}}{2 \pi} \int_{\mathbb{R}^{2}} e^{-\frac{x^{\prime} A x}{2}} e^{-x^{\prime} A x_{0}} \mathrm{~d} x .
$$

According to this equality,

$$
e^{h\left(S_{1}, S_{2}\right)}=e^{\frac{S^{\prime} J S}{2 N}}=c \int_{\mathbb{R}^{2}} e^{-\frac{1}{2 N} x^{\prime} J x} e^{-\frac{1}{N} x^{\prime} J S} \mathrm{~d} x,
$$

where $c=\frac{\sqrt{\operatorname{det} J}}{2 \pi}$.

We define the inverse matrix

$$
L=\left[\begin{array}{cc}
L_{1} & -\bar{L} \\
-\bar{L} & L_{2}
\end{array}\right]=\frac{1}{J_{1} J_{2}-\bar{J}^{2}}\left[\begin{array}{cc}
J_{2} & -\bar{J} \\
-\bar{J} & J_{1}
\end{array}\right]=J^{-1}
$$

eq: $\operatorname{defL}$

Switching variables $y=\frac{1}{N} J x$ we obtain 


$$
e^{h\left(S_{1}, S_{2}\right)}=c^{\prime} \int_{\mathbb{R}^{2}} e^{-\frac{N}{2} y \cdot L y} e^{S \cdot y} \mathrm{~d}^{2} y
$$

where $c^{\prime}$ is a term that depends on the matrix $L$ and on $N$. Equation ea our two-dimensional version of the Hubbard-Stratonovich transform.

Summing over all $(X, Y) \in\{-1,+1\}^{N}$ we obtain

$$
\begin{aligned}
\sum_{X, Y} e^{S \cdot y} & =\left(e^{+y_{1}}+e^{-y_{1}}\right)^{N_{1}} \cdot\left(e^{+y_{2}}+e^{-y_{2}}\right)^{N_{2}} \\
& =2^{N} \cosh ^{N_{1}}\left(y_{1}\right) \cdot \cosh ^{N_{2}}\left(y_{2}\right)
\end{aligned}
$$

and

$$
\begin{aligned}
& \sum_{X, Y} X_{1} \cdot \ldots X_{K} \cdot Y_{1} \cdot \ldots \cdot Y_{Q} e^{S \cdot y} \\
= & \frac{\left(e^{+y_{1}}-e^{-y_{1}}\right)^{K}}{\left(e^{+y_{1}}+e^{-y_{1}}\right)^{K}}\left(e^{+y_{1}}+e^{-y_{1}}\right)^{N_{1}} \cdot \frac{\left(e^{+y_{2}}-e^{-y_{2}}\right)^{Q}}{\left(e^{+y_{2}}+e^{-y_{2}}\right)^{Q}}\left(e^{+y_{2}}+e^{-y_{2}}\right)^{N_{2}} \\
= & 2^{N} \tanh ^{K}\left(y_{1}\right) \tanh ^{Q}\left(y_{2}\right) \cosh ^{N_{1}}\left(y_{1}\right) \cosh ^{N_{2}}\left(y_{2}\right)
\end{aligned}
$$

Consequently we have

$$
\begin{aligned}
& \sum_{X, Y} X_{1} \cdot \ldots X_{K} \cdot Y_{1} \cdot \ldots \cdot Y_{Q} e^{-H_{J}(X, Y)} \\
= & c \int e^{-N\left(1 / 2 y \cdot L y-N_{1} / N \ln \cosh y_{1}-N_{2} / N \ln \cosh y_{2}\right)} \tanh ^{K} y_{1} \tanh ^{Q} y_{2} \mathrm{~d}^{2} y \\
= & c \int e^{-N F_{J}(y)} \tanh ^{K} y_{1} \tanh ^{Q} y_{2} \mathrm{~d}^{2} y .
\end{aligned}
$$

where

$$
F_{J}(y):=\frac{1}{2} L_{1} y_{1}^{2}+\frac{1}{2} L_{2} y_{2}^{2}-\bar{L} y_{1} y_{2}-\alpha_{1} \ln \cosh y_{1}-\alpha_{2} \ln \cosh y_{2}
$$

Let us define

$$
Z_{J}(K, Q):=\int e^{-N F_{J}(y)} \tanh ^{K} y_{1} \tanh ^{Q} y_{2} \mathrm{~d}^{2} y
$$

then

$$
\mathbb{E}_{J}\left(X_{1} \cdot X_{2} \cdot \ldots \cdot X_{K} \cdot Y_{1} \cdot Y_{2} \cdot \ldots \cdot Y_{Q}\right)=\frac{Z_{J}(K, Q)}{Z_{J}(0,0)}
$$

Thus if we can compute $Z_{\text {lea }}\left(K_{\text {orrelations }} Q\right)$ asymptotically we will be able to compute the correlations (150). 


\subsection{Extrema of the function $F$}

\subsubsection{High Temperature Regime}

We are going to apply the Laplace method to evaluate the quantities $Z_{J}(K, Q)$. In order to do so we need to determine the minima of the function

$$
F\left(y_{1}, y_{2}\right)=\frac{1}{2} L_{1} y_{1}^{2}+\frac{1}{2} L_{2} y_{2}^{2}-\bar{L} y_{1} y_{2}-\alpha_{1} \ln \cosh y_{1}-\alpha_{2} \ln \cosh y_{2} .
$$

prop:pos-def Proposition 8. If

$$
\begin{aligned}
L_{1} & >\alpha_{1}, \\
\left(L_{1}-\alpha_{1}\right)\left(L_{2}-\alpha_{2}\right) & >\bar{L}^{2},
\end{aligned}
$$

then the function $F$ has a unique minimum at $(0,0)$.

$F$ has strictly positive definite Hessian

$$
H=\left[\begin{array}{ll}
F_{11} & F_{12} \\
F_{21} & F_{22}
\end{array}\right]
$$

and is therefore strictly convex.

We used $F_{i j}$ to denote the partial derivative of $F$ with respect to $y_{i}$ and $y_{j}$.

Remark 9. The conditions (133) and (1/4) are equivalent to the high temperature regime, as we shall show in proposition

Proof. We take derivatives with respect to both variables

$$
\begin{aligned}
F_{1}\left(y_{1}, y_{2}\right) & =L_{1} y_{1}-\bar{L} y_{2}-\alpha_{1} \tanh y_{1}, \\
F_{2}\left(y_{1}, y_{2}\right) & =L_{2} y_{2}-\bar{L} y_{1}-\alpha_{2} \tanh y_{2}, \\
F_{11}\left(y_{1}, y_{2}\right) & =L_{1}-\frac{\alpha_{1}}{\cosh ^{2} y_{1}}, \\
F_{22}\left(y_{1}, y_{2}\right) & =L_{2}-\frac{\alpha_{2}}{\cosh ^{2} y_{2}} .
\end{aligned}
$$

The Hessian matrix of $F$ is

$$
H=\left[\begin{array}{ll}
F_{11} & F_{12} \\
F_{21} & F_{22}
\end{array}\right]=\left[\begin{array}{cc}
L_{1}-\frac{\alpha_{1}}{\cosh ^{2} y_{1}} & -\bar{L} \\
-\bar{L} & L_{2}-\frac{\alpha_{2}}{\cosh ^{2} y_{2}}
\end{array}\right] .
$$

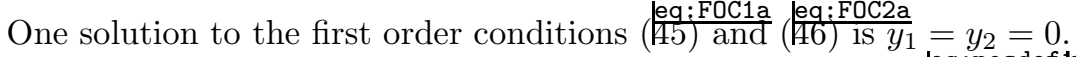

The matrix $H$ is positive definite at the origin if and only if leajposdef lea posdef 2 The matix $H$ is positive definite at the origin if and only if (14) Hence there is a local minimum at the origin. If the Hessian matrix at the origin is positive definite, it is also positive definite at any other point due to $\cosh |s|>\cosh |t|$ for all $|s|>|t|$. Thus $F$ is strictly convex and it follows that the minimum is unique and global. 
lem:det Lemma 10.

$$
\operatorname{sgn}\left(\operatorname{det}\left(\begin{array}{cc}
\frac{1}{\alpha_{1}}-J_{1} & \bar{J} \\
\bar{J} & \frac{1}{\alpha_{2}}-J_{2}
\end{array}\right)\right)=\operatorname{sgn}\left(\operatorname{det}\left(\begin{array}{cc}
L_{1}-\alpha_{1} & -\bar{L} \\
-\bar{L} & L_{2}-\alpha_{2}
\end{array}\right)\right)
$$

We used the notation

$$
\operatorname{sgn}(x)= \begin{cases}1, & \mathrm{x}>0 \\ 0, & \mathrm{x}=0 \\ -1, & \mathrm{x}<0\end{cases}
$$

Proof. We write

$$
\alpha^{-1}-J=J\left(J^{-1}-\alpha\right) \alpha^{-1}
$$

Since $\operatorname{det}(J), \operatorname{det}(\alpha)>0$ the assertion follows.

Proposition 11. The conditions on the Hessian matrix $H$ given in Proposition $\$$ are equivalent to the following conditions on the coupling matrix $J$ :

$$
\begin{aligned}
J_{1} & <\frac{1}{\alpha_{1}}, \\
J_{2} & <\frac{1}{\alpha_{2}}, \\
\bar{J}^{2} & <\left(\frac{1}{\alpha_{1}}-J_{1}\right)\left(\frac{1}{\alpha_{2}}-J_{2}\right) .
\end{aligned}
$$

eq: condJ2

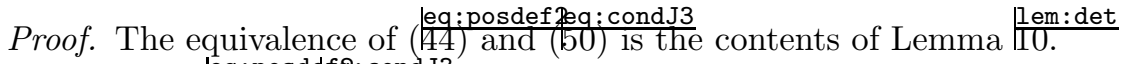

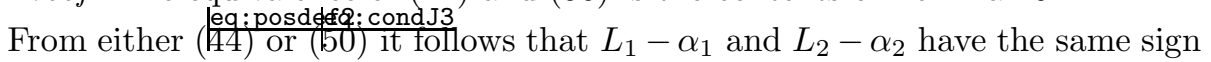
and also that $\frac{1}{\alpha_{1}}-J_{1}$ and $\frac{1}{\alpha_{2}}-J_{2}$ have the same sign.

A straight forward calculation shows

$$
\begin{aligned}
L_{1}>\alpha_{1} & \Longleftrightarrow J_{1}-\frac{1}{\alpha_{1}}<\frac{\bar{J}^{2}}{J_{2}} \\
\text { and } \quad L_{2}>\alpha_{2} & \Longleftrightarrow J_{2}-\frac{1}{\alpha_{2}}<\frac{\bar{J}^{2}}{J_{1}} .
\end{aligned}
$$

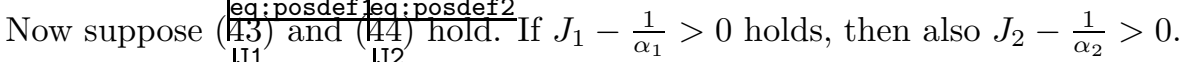
Consequently (151) and (52) imply that

$$
\left(J_{1}-\frac{1}{\alpha_{1}}\right)\left(J_{2}-\frac{1}{\alpha_{2}}\right)<\frac{\bar{J}^{4}}{J_{1} J_{2}}<\bar{J}^{2} .
$$

but this contradicts (50j). Thus $\frac{1}{\alpha_{1}}-J_{1}>0$.

If, on the other hand, $\frac{1}{\alpha_{1}}-J_{1}>0$ then (IJI) implies $L_{1}>\alpha_{1}$. 


\subsubsection{Critical regime}

We turn to the critical regime:

Proposition 12. If $L_{\nu}-\alpha_{\nu}>0$ for both groups and $\left(L_{1}-\alpha_{1}\right)\left(L_{2}-\alpha_{2}\right)=\bar{L}^{2}$, then the function $F$ defined in (142) has a unique global minimum at the origin.

Remark 13. The conditions stated in the proposition are equivalent to the critical regime This is shown in analogous fashion to the proof of Proposition III.

Proof. We take derivatives of $F$ with respect to both variables

$$
\begin{aligned}
& F_{1}\left(y_{1}, y_{2}\right)=L_{1} y_{1}-\bar{L} y_{2}-\alpha_{1} \tanh y_{1}=0 \\
& F_{2}\left(y_{1}, y_{2}\right)=L_{2} y_{2}-\bar{L} y_{1}-\alpha_{2} \tanh y_{2}=0
\end{aligned}
$$

One solution to this system of equations is $y_{1}=y_{2}=0$. We proceed to show that this solution is unique. We rewrite the function $F$ :

$$
F\left(t x_{0}, t y_{0}\right)=\frac{1}{2} L_{1} t^{2} x_{0}^{2}+\frac{1}{2} L_{2} t^{2} y_{0}^{2}-\bar{L} x_{0} y_{0} t^{2}-\alpha_{1} \ln \cosh t x_{0}-\alpha_{2} \ln \cosh t y_{0},
$$

where $\left(x_{0}, y_{0}\right)$ indicates the direction, $x_{0}^{2}+y_{0}^{2}=1$, and $t$ is the distance from the origin. The first derivative of $F$ with respect to $t$ is 0 at the origin, independently of the direction $\left(x_{0}, y_{0}\right)$.

We show that the second derivative $\frac{\mathrm{d}^{2} F\left(t x_{0}, t y_{0}\right)}{\mathrm{d} t^{2}}$ is positive in all directions, except for two.

$$
\frac{\mathrm{d}^{2} F\left(t x_{0}, t y_{0}\right)}{\mathrm{d} t^{2}}=L_{1} x_{0}^{2}+L_{2} y_{0}^{2}-2 \bar{L} x_{0} y_{0}-\frac{\alpha_{1} x_{0}^{2}}{\cosh ^{2} t x_{0}}-\frac{\alpha_{2} y_{0}^{2}}{\cosh ^{2} t y_{0}} .
$$

Therefore, we have

$$
\left.\frac{\mathrm{d}^{2} F\left(t x_{0}, t y_{0}\right)}{\mathrm{d} t^{2}}\right|_{t=0} \geq 0
$$

with equality if and only if both $t=0$ and

$$
\sqrt{L_{1}-\alpha_{1}} x_{0}-\sqrt{L_{2}-\alpha_{2}} y_{0}=0
$$

hold.

Hence there are two directions $\left(x_{0}, y_{0}\right)$, one pointing into quadrant one, the other into quadrant three, in which the second derivative is 0 at the origin. In all other directions the second derivative is strictly positive. For any direction, the second derivative is strictly positive for all $t>0$.

This concludes the proof that the minimum at the origin is unique and global. 


\subsubsection{Low Temperature Regime}

Assume we are in the low temperature regime, i.e. at least one of the following conditions holds:

$$
\begin{aligned}
J_{1} & >\frac{1}{\alpha_{1}} \\
J_{2} & >\frac{1}{\alpha_{2}} \\
\bar{J}^{2} & >\left(\frac{1}{\alpha_{1}}-J_{1}\right)\left(\frac{1}{\alpha_{2}}-J_{2}\right) .
\end{aligned}
$$

In terms of the inverse matrix $L=J^{-1}$, at least one of the following inequalities has to hold:

$$
\begin{aligned}
L_{1} & \leq \alpha_{1}, \\
L_{2} & \leq \alpha_{2}, \\
\left(L_{1}-\alpha_{1}\right)\left(L_{2}-\alpha_{2}\right) & <\bar{L}^{2} .
\end{aligned}
$$

In order to apply Laplace's method, we need to determine the minima of the function

$$
F(x, y)=\frac{1}{2} L_{1} x^{2}+\frac{1}{2} L_{2} y^{2}-\bar{L} x y-\alpha_{1} \ln \cosh x-\alpha_{2} \ln \cosh y .
$$

The first order conditions are

$$
\begin{aligned}
& F_{1}(x, y)=L_{1} x-\bar{L} y-\alpha_{1} \tanh x=0, \\
& F_{2}(x, y)=L_{2} y-\bar{L} x-\alpha_{2} \tanh y=0 .
\end{aligned}
$$

These equations always have a solution $(x, y)=(0,0)$. We define functions $X:[0, \infty) \rightarrow[0, \infty)$ and $Y:[0, \infty) \rightarrow[0, \infty)$ by setting $X(y)$ equal to the largest solution $x$ of equation (I51) given a value $y \geq 0$. Similarly, $Y(x)$ is defined as the largest solution $y$ of (52) given $x \geq 0$.

Proposition 14. The functions $X$ and $Y$ are strictly increasing and strictly concave.

Proof. We show the properties for $X$. By the implicit function theorem, we can calculate the first derivative of the function $X$ by dividing the partial derivative of the function

$$
G(x, y):=L_{1} x-\bar{L} y-\alpha_{1} \tanh x
$$

with respect to $x$ by the partial derivative of $G$ with respect to $y$. That yields 


$$
X^{\prime}(y)=\frac{\bar{L}}{L_{1}-\frac{\alpha_{1}}{\cosh ^{2} X(y)}} .
$$

We show that $X^{\prime}$ is always positive. We define two auxiliary functions

$$
\begin{aligned}
f, g & :[0, \infty) \rightarrow[0, \infty), \\
f(x) & :=L_{1} x-\bar{L} y, \\
g(x) & :=\alpha_{1} \tanh x .
\end{aligned}
$$

We are looking for the intersections of the functions $f$ and $g$ given a value of $y \geq 0$, the largest of which is precisely the value $X(y)$. If $y=0$ and $L_{1}-\alpha_{1} \geq 0, f$ and $g$ only intersect at 0 , so $X(0)=0$ in this case. If $L_{1}-\alpha_{1}<0$, or $y>0$ holds, then $f(0)=-\bar{L} y \leq 0, g(0)=0$, and $f^{\prime}(0)<g^{\prime}(0)$, so at the origin $f(0) \leq g(0)$, and for small values of $x f(x)<g(x)$. However, whereas $f^{\prime}(x)=L_{1}>0$ is constant, $g^{\prime}(x)=\frac{\alpha_{1}}{\cosh ^{2} x}>0$ is strictly decreasing in $x$ and $\lim _{x \rightarrow \infty} g^{\prime}(x)=0$. Therefore there is exactly one value $x_{1}>0$ such that $f\left(x_{1}\right)=g\left(x_{1}\right)$. Since for $x<x_{1} f(x)<g(x)$, it must be that $f^{\prime}\left(x_{1}\right)>g^{\prime}\left(x_{1}\right)$. This $x_{1}$ is $X(y)$. Hence we have

$$
L_{1}>\frac{\alpha_{1}}{\cosh ^{2} X(y)}
$$

and $X^{\prime}(y)>0$ has been shown.

The second derivative of $X$ is

$$
X^{\prime \prime}(y)=-\frac{2 \alpha_{1} \bar{L} X^{\prime}(y)}{\left(L_{1}-\frac{\alpha_{1}}{\cosh ^{2} X(y)}\right)^{2} \cosh ^{3} X(y)}<0,
$$

and so $X$ is strictly concave.

Proposition 15. The limits of the first derivatives of $X$ and $Y$ are

$$
\begin{aligned}
& \lim _{y \rightarrow \infty} X^{\prime}(y)=\frac{\bar{L}}{L_{1}}>0, \\
& \lim _{x \rightarrow \infty} Y^{\prime}(x)=\frac{\bar{L}}{L_{2}}>0 .
\end{aligned}
$$

Proof. We have

$$
\begin{aligned}
\lim _{y \rightarrow \infty} X^{\prime}(y) & =\frac{\bar{L}}{L_{1}-\frac{\alpha_{1}}{\cosh ^{2} X(y)}} \\
& =\frac{\bar{L}}{L_{1}}
\end{aligned}
$$


due to $\frac{\alpha_{1}}{\cosh ^{2} X(y)} \rightarrow 0$ as $X(y) \rightarrow \infty$. On the other hand, as $y$ goes to infinity, the solution $X(y)$ of (b1) has to go to infinity due to the boundedness of the term $\alpha_{1} \tanh x$.

\section{Corollary 16.}

$$
\lim _{y \rightarrow \infty} X^{\prime}(y) \lim _{x \rightarrow \infty} Y^{\prime}(x)=\frac{\bar{L}^{2}}{L_{1} L_{2}}<1
$$

holds.

Proof. By assumption, $J$, and therefore $L=J^{-1}$, are positive definite. In particular, the determinant of $L$ must be positive. Hence

$$
L_{1} L_{2}-\bar{L}^{2}>0
$$

We define the curves $\gamma_{1}, \gamma_{2}:[0, \infty) \rightarrow \mathbb{R}^{2}$ by setting

$$
\begin{aligned}
\gamma_{1}(y) & :=(X(y), y) \\
\gamma_{2}(x) & :=(x, Y(x)) .
\end{aligned}
$$

These curves originate at a certain point in the first quadrant that depends on the parameters $L_{1}, L_{2}, \alpha_{1}, \alpha_{2}$ :

$$
\begin{aligned}
\gamma_{1}(0) & := \begin{cases}(0,0), & L_{1}-\alpha_{1} \geq 0, \\
(X(0), 0), & L_{1}-\alpha_{1}<0,\end{cases} \\
\gamma_{2}(x): & = \begin{cases}(0,0), & L_{2}-\alpha_{2} \geq 0, \\
(0, Y(0)), & L_{2}-\alpha_{2}<0 .\end{cases}
\end{aligned}
$$

So curve $\gamma_{1}$ starts at the origin if and only if $L_{1}-\alpha_{1} \geq 0$. Similarly, $\gamma_{2}$ starts at the origin if and only if $L_{1}-\alpha_{1} \geq 0$.

Let us first assume the two curves do not meet at the origin. Then they start at points apart, but due to ([63), we have

$$
\lim _{x \rightarrow \infty} Y^{\prime}(x)<\frac{1}{\lim _{y \rightarrow \infty} X^{\prime}(y)} .
$$

This implies the curves have to meet at some point in the interior of the first quadrant. Call this point $\left(x_{1}, y_{1}\right)$. Once they have met, the strict concavity of both $X$ and $Y$ drives them apart and they do not intersect again. Hence the point $\left(x_{1}, y_{1}\right)$ is uniquely determined.

If the two curves do meet at the origin, we have $L_{1}-\alpha_{1} \geq 0$ and $L_{2}-\alpha_{2} \geq 0$. We distinguish the two cases 
1. $L_{1}-\alpha_{1}>0$ and $L_{2}-\alpha_{2}>0$,

2. $L_{1}-\alpha_{1}=0$ or $L_{2}-\alpha_{2}=0$.

In the first case, since we are in the low temperature regime,

$$
\left(L_{1}-\alpha_{1}\right)\left(L_{2}-\alpha_{2}\right)<\bar{L}^{2}
$$

must hold, and we have

$$
\begin{aligned}
X^{\prime}(0) Y^{\prime}(0) & =\frac{\bar{L}}{L_{1}-\alpha_{1}} \frac{\bar{L}}{L_{2}-\alpha_{2}} \\
& >1 .
\end{aligned}
$$

So

$$
Y^{\prime}(0)>\frac{1}{X^{\prime}(0)},
$$

which means that the two curves starting at $(0,0)$ initially move apart. So for some points close to the origin, the curves are apart, and the previous reasoning for the existence of a unique point of intersection inside the first quadrant applies.

In the second case, if $L_{1}-\alpha_{1}=0$, then the derivative of function $X$ is infinite at the origin, meaning $\gamma_{1}$ moves parallel to the x-axis. The function $Y$ on the other hand either has positive or infinite derivative at the origin, so $\gamma_{2}$ either moves into the interior of the first quadrant or it moves parallel to the $y$-axis. In any case, the two curves move apart after leaving the origin.

We summarize:

thm:corrl Theorem 17. In the low temperature regime the function $F$ has exactly two minima $\mu^{*}=\left(\mu_{1}^{*}, \mu_{2}^{*}\right)$ and $-\mu^{*}$ and we may suppose that $\mu_{1}^{*}, \mu_{2}^{*}>0$.

sub: corrhigh

\subsection{Correlations for the high temperature regime}

In this section we use the Laplace method to evaluate the expression $Z_{J}(K, Q)$ and thus the correlation (130) asymptotically in the high temperature regime.

Let $H=J^{-1}-\left(\begin{array}{cc}\alpha_{1} & 0 \\ 0 & \alpha_{2}\end{array}\right)$ be the Hessian of $F$ at $0, \mathcal{N}\left(0, H^{-1}\right)$ the twodimensional normal distribution with covariance matrix $H^{-1}$ and let

$$
\begin{aligned}
\nu_{K, Q} & =\nu_{K, Q}\left(0, H^{-1}\right) \\
& =\frac{\sqrt{\operatorname{det} H}}{2 \pi} \int_{\mathbb{R}^{2}} e^{-\frac{1}{2}\left(\begin{array}{ll}
x_{1} & x_{2}
\end{array}\right) H\left(\begin{array}{l}
x_{1} \\
x_{2}
\end{array}\right)}{ }_{x_{1}{ }^{K}} x_{2}{ }^{Q} d x_{1} d x_{2}
\end{aligned}
$$


be the moments of $\mathcal{N}\left(0, H^{-1}\right)$.

In the following proposition as in the whole paper by $a_{N} \approx b_{N}$ we mean $\lim _{N \rightarrow \infty} \frac{a_{N}}{b_{N}}=1$.

prop:ZJhigh Proposition 18. Let $J, \alpha_{1}, \alpha_{2}$ satisfy (113)-(115) (high temperature regime), then

$$
\begin{aligned}
Z_{J}(K, Q) & =\int e^{-N F_{J}(y)} \tanh ^{K} y_{1} \tanh ^{Q} y_{2} \mathrm{~d}^{2} y \\
& \approx \frac{2 \pi}{\sqrt{\operatorname{det} H}} \nu_{K, Q}\left(0, H^{-1}\right) N^{-\frac{K+Q}{2}-1} \quad \text { as } N \rightarrow \infty .
\end{aligned}
$$

Proof. We use the Laplace method to evaluate $Z_{J}(K, Q)$. We only sketch the main idea. For the details, in particular the remainder estimates, we refer to [19. or [14. In the integral we replace both $F$ and the tanh terms by the leading terms in their Taylor expansion around 0 . This gives

$$
\begin{aligned}
Z_{J}(K, Q) & \approx \int e^{-N \cdot 1 / 2\left(y^{\prime} H y\right)} y_{1}^{K} y_{2}^{Q} d y_{1} d y_{2} \\
& =N^{-\frac{K+Q}{2}-1} \int e^{-\frac{1}{2}\left(x^{\prime} H x\right)} x_{1}^{K} x_{2}^{Q} d x_{1} d x_{2} \\
& =\frac{2 \pi}{\sqrt{\operatorname{det} H}} \nu_{K, Q}\left(H^{-1}\right) N^{-\frac{K+Q}{2}-1}
\end{aligned}
$$

where we changed variable $x=\sqrt{N} y$.

Proposition lorop:ZJhigh 1 immediately gives:

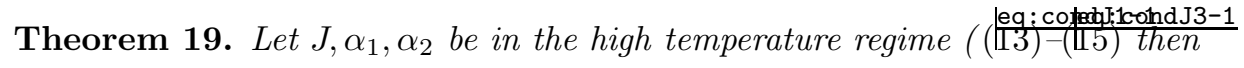

$$
\mathbb{E}\left(X_{1} \cdot X_{2} \cdot \ldots \cdot X_{K} \cdot Y_{1} \cdot Y_{2} \cdot \ldots \cdot Y_{Q}\right) \approx \nu_{K, Q}\left(0, H^{-1}\right) N^{-(K+Q) / 2}
$$

\subsection{Correlations for the critical regime}

Expanding again $F$ to leading order gives in the critical regime

$$
\begin{aligned}
F\left(y_{1}, y_{2}\right) & \approx \frac{1}{2}\left(\left(L_{1}-\alpha_{1}\right) y_{1}^{2}+\left(L_{2}-\alpha_{2}\right) y_{2}^{2}-2 \bar{L} y_{1} y_{2}+\frac{2 \alpha_{1}}{12} y_{1}^{4}+\frac{2 \alpha_{2}}{12} y_{2}^{4}\right) \\
& =\frac{1}{2}\left(\left(\sqrt{L_{1}-\alpha_{1}} y_{1}-\sqrt{L_{2}-\alpha_{2}} y_{2}\right)^{2}+\frac{\alpha_{1}}{6} y_{1}^{4}+\frac{\alpha_{2}}{6} y_{2}^{4}\right)
\end{aligned}
$$

Thus we have

$$
Z_{J}(K, Q) \approx \int_{\mathbb{R}^{2}} e^{-N / 2\left(\left(\sqrt{L_{1}-\alpha_{1}} y_{1}-\sqrt{L_{2}-\alpha_{2}} y_{2}\right)^{2}+\frac{\alpha_{1}}{6} y_{1}^{4}+\frac{\alpha_{2}}{6} y_{2}^{4}\right)} y_{1}^{K} y_{2}^{Q} d y_{1} d y_{2}
$$

We substitute 


$$
\begin{aligned}
& u=N^{1 / 2}\left(\sqrt{L_{1}-\alpha_{1}} y_{1}-\sqrt{L_{2}-\alpha_{2}} y_{2}\right) \\
& v=N^{1 / 4}\left(\sqrt{L_{1}-\alpha_{1}} y_{1}+\sqrt{L_{2}-\alpha_{2}} y_{2}\right)
\end{aligned}
$$

which gives

$$
\int_{\mathbb{R}^{2}} e^{-\frac{1}{2}\left[u^{\prime 2}+\frac{\alpha_{1}}{2^{5} \cdot 3\left(L_{1}-\alpha_{1}\right)^{2}}\left(\frac{u^{\prime}}{N^{1 / 4}}+v^{\prime}\right)^{4}+\frac{\alpha_{2}}{2^{5} \cdot 3\left(L_{2}-\alpha_{2}\right)^{2}}\left(v^{\prime}-\frac{u^{\prime}}{N^{1 / 4}}\right)^{4}\right]}\left(\frac{u^{\prime}}{N^{1 / 2}}+\frac{v^{\prime}}{N^{1 / 4}}\right)^{K}\left(\frac{v^{\prime}}{N^{1 / 4}}-\frac{u^{\prime}}{N^{1 / 2}}\right)^{L} \mathrm{~d} u^{\prime} \mathrm{d} v^{\prime}
$$

times a constant equal to

$$
\frac{1}{2^{K+L+1}\left(L_{1}-\alpha_{1}\right)^{\frac{K+1}{2}}\left(L_{2}-\alpha_{2}\right)^{\frac{L+1}{2}} N^{\frac{3}{4}}} .
$$

Since we are interested merely in the ratio $\frac{Z_{J}(K, Q)}{Z_{J}(0,0)}$ we may (and will) neglect multiplicative constants in the evaluation of $Z_{J}(K, Q)$ as long as these constants are independent of $K$ and $Q$. To shorten notation we define

$$
a_{N}(K, Q) \sim b_{N}(K, Q) \quad \text { if } \quad \lim _{N \rightarrow \infty} \frac{a_{N}}{b_{N}} \rightarrow c
$$

for a constant $0<c<\infty$ which is independent of $K$ and $Q$. With this notation we have

$$
\begin{aligned}
Z_{J}(K, Q) & \sim \int_{\mathbb{R}^{2}} e^{-1 / 2 u^{2}} e^{-1 /(3 \cdot 26)\left(\frac{\alpha_{1}}{\left(L_{1}-\alpha_{1}\right)^{2}}+\frac{\alpha_{2}}{\left.\left(L_{2}-\alpha_{2}\right)^{2}\right)} v^{4}\right)} v^{K+Q} d u d v \\
& \sim \int e^{-1 /(3 \cdot 26)\left(\frac{\alpha_{1}}{\left(L_{1}-\alpha_{1}\right)^{2}}+\frac{\alpha_{2}}{\left.\left(L_{2}-\alpha_{2}\right)^{2}\right)} v^{4}\right)} v^{K+Q} d v
\end{aligned}
$$

for constants $c, c^{\prime}$ independent of $K$ and $Q$. We note that

$$
\int_{0}^{\infty} e^{-a \cdot x^{4}} x^{m} d x=\frac{1}{4 a^{\frac{m+1}{4}}} \Gamma\left(\frac{m+1}{4}\right)
$$

Summing up, we obtain

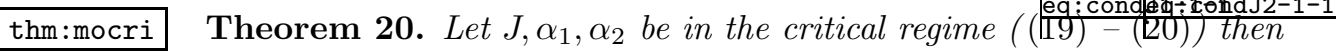

$$
\begin{gathered}
\mathbb{E}\left(X_{1} \cdot \ldots \cdot X_{K} \cdot Y_{1} \cdot \ldots \cdot Y_{Q}\right) \approx\left[\frac{12}{\alpha_{1}\left(L_{2}-\alpha_{2}\right)^{2}+\alpha_{2}\left(L_{1}-\alpha_{1}\right)^{2}}\right]^{\frac{K+Q}{4}} \\
\cdot\left(L_{1}-\alpha_{1}\right)^{Q / 2}\left(L_{2}-\alpha_{2}\right)^{K / 2} \frac{\Gamma\left(\frac{K+Q+1}{4}\right)}{\Gamma\left(\frac{1}{4}\right)} \cdot N^{-\frac{K+Q}{4}}
\end{gathered}
$$




\subsection{Correlations for the Low Temperature Regime}

Using again Laplace's method to evaluate the expressions for $Z_{J}(K, Q)$ for the low temperature regime we immediately get:

Theorem 21. In the low temperature regime we have

$\left.\mathbb{E}\left(X_{1} \cdot \ldots \cdot X_{K} \cdot Y_{1} \cdot \ldots \cdot Y_{Q}\right)\right) \approx \begin{cases}\tanh ^{K}\left(\mu_{1}\right) \tanh ^{Q}\left(\mu_{2}\right), & \text { if } K+Q \text { is even } \\ 0, & \text { otherwise. }\end{cases}$

where $\mu_{*}=\left(\mu_{1}^{*}, \mu_{2}^{*}\right)$ is given in Theorem $t$ thp: corrl

\section{Laws of large numbers}

sec:lln

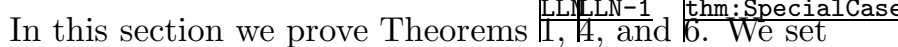

$$
W_{K, N_{1}}:=\left\{1,2, \ldots, N_{1}\right\}^{K}
$$

We also denote by $W_{K, N_{1}}(r)$ the set of all multiindices $\underline{i}=\left(i_{1}, i_{2}, \ldots, i_{K}\right) \in$ $W_{K, N_{1}}$ for which exactly $r$ indices occur only once and by $w_{K, N_{1}}(r)$ the cardinality of $W_{K, N_{1}}(r)$.

We have

Lemma 22.

$$
w_{K, N_{1}}(r) \leq K ! N_{1}^{\frac{K+r}{2}}
$$

Proof. The multiindices in $W_{K, N_{1}}(r)$ contain at most $r+\frac{K-r}{2}=\frac{K+r}{2}$ different indices. There are at most $N_{1}^{\frac{K+r}{2}}$ ways to choose them and at most $K$ ! ways to order them.

thm:llnmoments

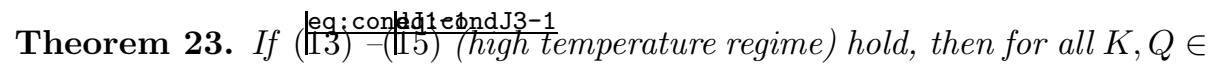
$\mathbb{N}, K, Q>0$

$$
\mathbb{E}\left(\left(\frac{1}{N_{1}} \sum_{i=1}^{N_{1}} X_{i}\right)^{K}\left(\frac{1}{N_{2}} \sum_{j=1}^{N_{2}} Y_{j}\right)^{Q}\right) \rightarrow 0
$$

eq: 1 ln-moment

lea:lln-moment

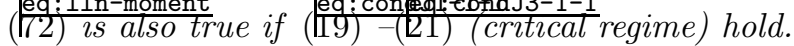


Proof.

$$
\begin{aligned}
& \mathbb{E}\left(\left(\frac{1}{N_{1}} \sum_{i=1}^{N_{1}} X_{i}\right)^{K}\left(\frac{1}{N_{2}} \sum_{j=1}^{N_{2}} Y_{j}\right)^{Q}\right) \\
= & \frac{1}{N_{1}^{K} N_{2}^{Q}} \sum_{\underline{i} \in W_{K, N_{1}}} \sum_{\underline{j} \in W_{Q}, N_{2}} \mathbb{E}\left(X_{i_{1}} \cdot X_{i_{2}} \cdot \ldots \cdot X_{i_{K}} Y_{j_{1}} \cdot Y_{j_{2}} \cdot \ldots \cdot Y_{j_{Q}}\right) \\
= & \frac{1}{N_{1}^{K} N_{2}^{Q}} \sum_{k=0}^{K} \sum_{q=0}^{Q} \sum_{\underline{i} \in W_{K, N_{1}}(k)} \sum_{\underline{j} \in W_{Q, N_{2}}(q)} \mathbb{E}\left(X_{i_{1}} \cdot X_{i_{2}} \cdot \ldots \cdot X_{i_{K}} Y_{j_{1}} \cdot Y_{j_{2}} \cdot \ldots \cdot Y_{j_{Q}}\right) \\
\leq & C \frac{1}{N_{1}^{K} N_{2}^{Q}} N_{1}^{\frac{K+k}{2}} N_{2}^{\frac{Q+q}{2}} N^{-(k+q) / 2} \rightarrow 0
\end{aligned}
$$

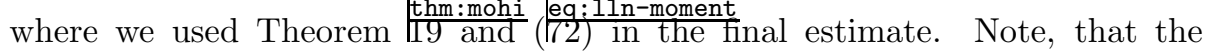
constant $C$ depends on $K$ and $Q$, but not on $N$.

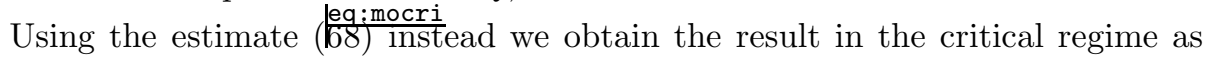
well.

We also note that the estimates hold in the cases $\alpha_{1}=0$ or $\alpha_{2}=0$.

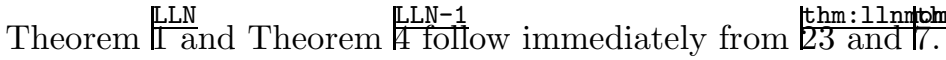

Remark 24. We have actually proved that

$$
\mathbb{E}\left(\left(\frac{1}{N_{1}^{\gamma}} \sum_{i=1}^{N_{1}} X_{i}\right)^{K}\left(\frac{1}{N_{2}^{\gamma}} \sum_{j=1}^{N_{2}} Y_{j}\right)^{Q}\right)
$$

is bounded with $\gamma=\frac{1}{2}$ in the high temperature regimeand $\gamma \overline{\overline{1} \text { uct }} \frac{3}{\text { in }}$ the the critical regime. This is an indication that the limit theorems 遂 and 递 may hold.

We turn to the low temperature regime. Analogous to $\left(\frac{\text { leq }}{113}\right)$ we obtain

$$
\begin{aligned}
& \mathbb{E}\left(\left(\frac{1}{N_{1}} \sum_{i=1}^{N_{1}} X_{i}\right)^{K}\left(\frac{1}{N_{2}} \sum_{j=1}^{N_{2}} Y_{j}\right)^{Q}\right) \\
= & \frac{1}{N_{1}^{K} N_{2}^{Q}} \sum_{k=0}^{K} \sum_{q=0}^{Q} \sum_{\underline{i} \in W_{K, N_{1}}(k)} \sum_{\underline{j} \in W_{Q, N_{2}}(q)} \mathbb{E}\left(X_{i_{1}} \cdot X_{i_{2}} \cdot \ldots \cdot X_{i_{K}} Y_{j_{1}} \cdot Y_{j_{2}} \cdot \ldots \cdot Y_{j_{Q}}\right)
\end{aligned}
$$

The terms with $k<K$ and $q<Q$ are canceled by the term in front of the sum. Thus (IIT) is asymptotically given by

$$
\begin{aligned}
& \mathbb{E}\left(X_{1} \cdot X_{2} \cdot \ldots \cdot X_{K} Y_{1} \cdot Y_{2} \cdot \ldots \cdot Y_{Q}\right) \\
\approx & \frac{1}{2}\left(1+(-1)^{K+Q}\right) \tanh ^{K}\left(\mu_{1}^{*}\right) \cdot \tanh ^{Q}\left(\mu_{2}^{*}\right)
\end{aligned}
$$




\section{The Central Limit Theorem}

subs : comb

\subsection{Some Combinatorics}

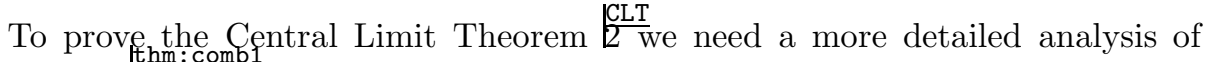
Lemma

Let us define $W_{K, N_{1}}^{0}(r)$ to be the set of all multiindices $\underline{i}=\left(i_{1}, i_{2}, \ldots, i_{K}\right) \in$ $W_{K, N_{1}}(r)$ for which no index occurs more than twice. We also set

$$
W_{K, N_{1}}^{+}(r):=W_{K, N_{1}}(r) \backslash W_{K, N_{1}}^{0}(r)
$$

and denote by $w_{K, N_{1}}^{+}(r)$ and $w_{K, N_{1}}^{0}(r)$ their cardinalities.

lem:comb2 Lemma 25.

$$
w_{K, N_{1}}^{+}(r) \leq K ! N_{1}^{\frac{K+r}{2}-\frac{1}{2}}
$$

Proof. If the $K$-tuple $\underline{i}$ contains $r$ indices with only one occurrence and at least one index with three or more occurrences there are at most $r-3$ places left for indices with (exactly) two occurrences. Therefore, a tuple in $w_{K, N_{1}}^{+}(r)$ contains at most $k+1+\frac{K-r-3}{2}$ different indices. Consequently there are at most $K ! N_{1}^{\frac{K+r}{2}-\frac{1}{2}}$ such tuples.

lem: comb4 Lemma 26.

$$
w_{K, N_{1}}^{0}(r)= \begin{cases}\frac{N_{1} !}{\left(N_{1}-\frac{K+r}{2}\right) !} \frac{K !}{r !\left(\frac{K-r}{2}\right) ! 2^{\frac{K-r}{2}},}, & \text { if } K-r \text { is even } \\ 0, & \text { else. }\end{cases}
$$

Proof. We choose an (ordered) $r$-tuple $\rho$ of $r$ indices to occur once and an ordered $(K-r) / 2$-tuple $\lambda$ of indices to occur twice in $\underline{i}$. We have

$$
\frac{N_{1} !}{\left(N_{1}-\frac{K+r}{2}\right) !}
$$

ways to do so.

Then we choose the $r$ positions for those indices which occur once. We can do this in

$$
\left(\begin{array}{c}
K \\
r
\end{array}\right)=\frac{K !}{r !(K-r) !}
$$

ways. We fill these positions in $\underline{i}$ with $\rho_{1}, \rho_{2}, \ldots, \rho_{r}$ starting with the left most open position.

Finally we distribute the indices $\lambda_{1}, \ldots, \lambda_{(K-r) / 2}$, twice each. The index $\lambda_{1}$ is put at the left most free place in $\underline{i}$ and in one of the remaining $K-r-1$ positions, $\lambda_{2}$ is put at the then first free place in $\underline{i}$ and in one of the $K-r-3$ remaining free places and so on. 
This gives

$$
(K-r-1) ! !=\frac{(K-r) !}{\left(\frac{K-r}{2}\right) ! 2^{\frac{K-r}{2}}}
$$

possibilities.

We summarize the above considerations in the following Theorem.

thm:comb3 Theorem 27. In the high temperature regime we have

$$
\begin{aligned}
& \mathbb{E}\left(\left(\frac{1}{N_{1}^{\frac{1}{2}}} \sum_{i=1}^{N_{1}} X_{i}\right)^{2 K}\left(\frac{1}{N_{2}^{\frac{1}{2}}} \sum_{j=1}^{N_{2}} Y_{j}\right)^{2 Q}\right) \\
\approx & \sum_{k=0}^{K} \sum_{q=0}^{Q} \frac{(2 K) ! \alpha_{1}^{k}}{(2 k) !(K-k) ! 2^{K-k}} \frac{(2 Q) ! \alpha_{2}^{q}}{(2 q) !(Q-q) ! 2^{Q-q}} \nu_{2 k, 2 q}\left(0, H^{-1}\right)
\end{aligned}
$$

and

$$
\begin{aligned}
& \mathbb{E}\left(\left(\frac{1}{N_{1}^{\frac{1}{2}}} \sum_{i=1}^{N_{1}} X_{i}\right)^{2 K+1}\left(\frac{1}{N_{2}^{\frac{1}{2}}} \sum_{j=1}^{N_{2}} Y_{j}\right)^{2 Q+1}\right) \\
\approx & \sum_{k=0}^{K} \sum_{q=0}^{Q} \frac{(2 K+1) ! \alpha_{1}^{k+\frac{1}{2}}}{(2 k+1) !(K-k) ! 2^{K-k}} \frac{(2 Q+1) ! \alpha_{2}^{q+\frac{1}{2}}}{(2 q+1) !(Q-q) ! 2^{Q-q}} \nu_{2 k+1,2 q+1}\left(0, H^{-1}\right)
\end{aligned}
$$

\subsection{Moments of a 2d-normal distribution}

Let $\Sigma=\left(\begin{array}{cc}\sigma_{1} & \bar{\sigma} \\ \bar{\sigma} & \sigma_{2}\end{array}\right)$ be a covariance matrix and let $\left(\begin{array}{c}Z_{1} \\ Z_{2}\end{array}\right) \sim \mathcal{N}(0, \Sigma)$ distributed.

We write $\nu_{K, Q}(\Sigma)=\mathbb{E}\left(Z_{1}^{K} Z_{2}^{Q}\right)$ to denote the moment of $\mathcal{N}(0, \Sigma)$ of order $(K, Q)$.

\section{Proposition 28.}

$\nu_{2 K, 2 Q}(\Sigma)=\sum_{r=0}^{K \wedge Q} \frac{2 K !}{(2 K-2 r) ! 2 r !} \frac{2 Q !}{(2 Q-2 r) !} \frac{(2 K-2 r) !}{(K-r) ! 2^{K-r}} \frac{(2 Q-2 r) !}{(Q-r) ! 2^{Q-r}} \sigma_{1}^{K-r} \bar{\sigma}^{2 r} \sigma_{2}^{Q-r}$

Proof. Suppose $V_{1}, \ldots, V_{2 n}$ are random variables. Denote by $\mathcal{P}_{2}=\mathcal{P}_{2}(2 n)$ the set of pair partitions of $1, \ldots, 2 n$. For a pair partition $\pi=\left\{\pi_{1}, \ldots, \pi_{n}\right\} \in \mathcal{P}_{2}$ we set $\prod_{\pi}\left(V_{1}, \ldots, V_{2 n}\right)=\prod_{i=1}^{n} \mathbb{E}\left(V^{\pi_{i}}\right)$ where $V^{\{i, j\}}=V_{i} \cdot V_{j}$. 
By Isserlis' theorem lisserlis

$$
\begin{aligned}
\nu_{2 K, 2 Q}(\Sigma) & =\sum_{\pi \in \mathcal{P}_{2}(2 K+2 Q} \Pi_{\pi}(\underbrace{Z_{1}, Z_{1}, \ldots, Z_{1}}_{2 K \text { times }} \underbrace{Z_{2}, Z_{2}, \ldots, Z_{2}}_{2 Q \text { times }}) \\
& =\sum_{r=0}^{K \wedge Q} \rho_{r} \cdot \sigma_{1}^{2 K-2 r} \bar{\sigma}^{2 r} \sigma_{2}^{2 Q-2 r}
\end{aligned}
$$

where $\rho_{r}=\#\{\pi \mid \pi$ contains exactly $2 r$ mixed pairs $\}$. (Mixed pairs are of the form $\{i, j\}$ with $i \leq 2 K$ and $j>2 K$.)

To compute $\rho_{r}$ we first choose $2 r$ " $Z_{1}$ 's". This can be done in $\left(\begin{array}{c}2 K \\ 2 r\end{array}\right)$ ways. For these $Z_{1}$ 's choose $2 r Z_{2}$ 's : $\frac{(2 L) !}{(2 L-2 r) !}$. This gives $\left(\begin{array}{c}2 K \\ 2 r\end{array}\right) \frac{(2 L) !}{(2 L-2 r) !}$. The remaining terms come from the pair partitions of the $2 K-2 r Z_{1}$ 's and $2 Q-2 R$ $Z_{2}$ 's.

sub:prclt

\subsection{Proof of Theorem $[2$}

We calculate the moments $\mathbb{E}\left(\left(\frac{1}{N_{1}^{1 / 2}} \sum X_{i}\right)^{2 K}\left(\frac{1}{N_{2}^{1 / 2}} \sum Y_{j}\right)^{2 Q}\right)$, i. e. those with even exponents. The case of odd exponents is done in a similar way.

We write $H^{-1}=\left(\begin{array}{cc}\sigma_{1} & \bar{\sigma} \\ \bar{\sigma} & \sigma_{2}\end{array}\right)$

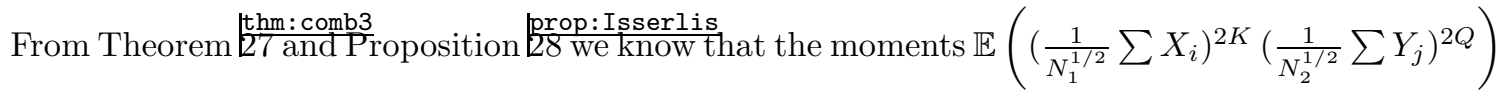
are approximately given by 


$$
\begin{aligned}
& \sum_{k=0}^{K} \sum_{l=0}^{Q} \frac{2 K ! \alpha_{1}^{k}}{2 k !(K-k) ! 2^{K-k}} \frac{2 Q ! \alpha_{2}^{l}}{2 l !(Q-l) ! 2^{Q-l}} \nu_{k, l}\left(H^{-1}\right) \\
& =\sum_{k=0}^{K} \sum_{l=0}^{Q} \frac{2 K ! \alpha_{1}^{k}}{2 k !(K-k) ! 2^{K-k}} \frac{2 Q ! \alpha_{2}^{l}}{2 l !(Q-l) ! 2^{Q-l}} \\
& \cdot \sum_{r=0}^{k \wedge l} \frac{2 k !}{(2 k-2 r) ! 2 r !} \frac{2 l !}{(2 l-2 r) !} \frac{(2 k-2 r) !}{(k-r) ! 2^{k-r}} \frac{(2 l-2 r) !}{(l-r) ! 2^{l-r}} \sigma_{1}^{k-r} \bar{\sigma}^{2 r} \sigma_{2}^{l-r} \\
& =\sum_{r=0}^{K \wedge Q} \sum_{k=r}^{K} \sum_{l=r}^{Q} \frac{2 K ! \alpha_{1}^{k}}{(K-k) ! 2^{K-k}} \frac{2 Q ! \alpha_{2}^{l}}{(Q-l) ! 2^{Q-l}} \frac{2^{2 r}}{2 r !(k-r) !(l-r) ! 2^{k} 2^{l}} \sigma_{1}^{k-r} \bar{\sigma}^{2 r} \sigma_{2}^{l-r} \\
& =\sum_{r=0}^{K \wedge Q} \sum_{k=r}^{K} \sum_{l=r}^{Q} \frac{2 K ! \alpha_{1}^{k}}{(K-k) ! 2^{K-r}} \frac{2 Q ! \alpha_{2}^{l}}{(Q-l) ! 2^{Q-r}} \frac{1}{2 r !(k-r) !(l-r) !} \sigma_{1}^{k-r} \bar{\sigma}^{2 r} \sigma_{2}^{l-r} \\
& \text { Setting } s=k-r, t=l-r \text { (i.e. } k=s+r, l=t+r \text { ) gives: } \\
& =\sum_{r=0}^{K \wedge Q} \sum_{s=0}^{K-r} \sum_{t=0}^{Q-r} \frac{2 K ! \alpha_{1}^{s+r}}{(K-r-s) ! 2^{K-r}} \frac{2 Q ! \alpha_{2}^{t+r}}{(Q-r-s) ! 2^{Q-r}} \frac{1}{2 r ! s ! t !} \sigma_{1}^{s} \bar{\sigma}^{2 r} \sigma_{2}^{t} \\
& =\sum_{r=0}^{K \wedge l} \frac{2 K !}{(2 K-2 r) ! 2 r !} \frac{2 Q !}{(2 Q-2 r) !} \frac{(2 K-2 r) !}{(K-r) ! 2^{K-r}} \frac{(2 Q-2 r) !}{(Q-r) ! 2^{Q-r}}\left(\sqrt{\alpha_{1} \alpha_{2}} \bar{\sigma}\right)^{2 r} \\
& \cdot \sum_{s=0}^{K-r} \frac{(K-r) !}{(K-r-s) ! s !}\left(\alpha_{1} \sigma_{1}\right)^{s} \cdot \sum_{t=0}^{Q-r} \frac{(Q-r) !}{(Q-r-s) ! t !}\left(\alpha_{2} \sigma_{2}\right)^{t} \\
& =\sum_{r=0}^{K \wedge Q} \frac{2 K !}{(2 K-2 r) ! 2 r !} \frac{2 Q !}{(2 Q-2 r) !} \frac{(2 K-2 r) !}{(K-r) ! 2^{K-r}} \frac{(2 Q-2 r) !}{(Q-r) ! 2^{Q-r}}\left(1+\alpha_{1} \sigma_{1}\right)^{K-r}\left(\sqrt{\alpha_{1} \alpha_{2}} \bar{\sigma}\right)^{2 r}\left(1+\alpha_{2} \sigma_{2}\right)^{Q-r} \\
& =\nu_{2 K, 2 Q}(C)
\end{aligned}
$$

\subsection{Proof of Theorem Fluctuations}

Similar to the high temperature regime we evaluate

$$
\begin{aligned}
& \mathbb{E}\left(\left(\frac{1}{N_{1}^{\frac{3}{4}}} \sum_{i=1}^{N_{1}} X_{i}\right)^{K}\left(\frac{1}{N_{2}^{\frac{3}{4}}} \sum_{j=1}^{N_{2}} Y_{j}\right)^{Q}\right) \\
= & \frac{1}{N_{1}^{\frac{3}{4}} K N_{2}^{\frac{3}{4}} Q} \sum_{k=0}^{K} \sum_{q=0}^{Q} w_{K, N_{1}}^{0}(k) w_{Q, N_{2}}^{0}(q) \mathbb{E}\left(X_{1} \ldots X_{k} \cdot Y_{1} \ldots Y_{q}\right)
\end{aligned}
$$


By Theorem [u] and Lecri

$$
\frac{1}{N_{1}^{\frac{3}{4} K} N_{2}^{\frac{3}{4} Q}} w_{K, N_{1}}^{0}(k) w_{Q, N_{2}}^{0}(q) \mathbb{E}\left(X_{1} \ldots X_{k} \cdot Y_{1} \ldots Y_{q}\right) \leq C N^{-\frac{K-k}{4}-\frac{Q-q}{4}}
$$

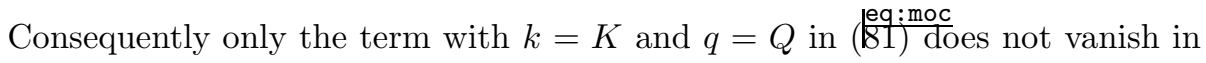
the large- $N$-limit. Thus

$$
\begin{aligned}
& \mathbb{E}\left(\left(\frac{1}{N_{1}^{\frac{3}{4}}} \sum_{i=1}^{N_{1}} X_{i}\right)^{K}\left(\frac{1}{N_{2}^{\frac{3}{4}}} \sum_{j=1}^{N_{2}} Y_{j}\right)^{Q}\right) \approx \alpha_{1}^{\frac{K}{4}} \alpha_{2}^{\frac{Q}{4}} N^{\frac{K}{4}+\frac{Q}{4}} \mathbb{E}\left(X_{1} \cdot \ldots \cdot X_{K} \cdot Y_{1} \cdot \ldots \cdot Y_{Q}\right) \\
\approx & \alpha_{1}^{\frac{K}{4}} \alpha_{2}^{\frac{Q}{4}}\left[\frac{12}{\alpha_{1}\left(L_{2}-\alpha_{2}\right)^{2}+\alpha_{2}\left(L_{1}-\alpha_{1}\right)^{2}}\right]^{\frac{K+Q}{4}} \cdot\left(L_{1}-\alpha_{1}\right)^{Q / 2}\left(L_{2}-\alpha_{2}\right)^{K / 2} \frac{\Gamma\left(\frac{K+Q+1}{4}\right)}{\Gamma\left(\frac{1}{4}\right)}
\end{aligned}
$$

\section{References}

[1] Breiman, Leo: Probability, Addison-Wesley 1968

[2] Collet, F.: Macroscopic Limit of a Bipartite Curie-Weiss Model: A Dynamical Approach, J. Stat. Phys. 157(6), pp. 1301-1319 (2014)

[3] Contucci, Pierluigi, Gallo, Ignacio: Bipartite Mean Field Spin Systems. Existence and Solution, Math. Phys. Elec. Jou. Vol 14, N.1, 1-22 (2008)

[4] Contucci, Pierluigi and Ghirlanda, S.: Modelling Society with Statistical Mechanics: an Application to Cultural Contact and Immigration. Quality and Quantity, 41, 569-578 (2007)

[5] Ellis, Richard: Entropy, large deviations, and statistical mechanics, Whiley 1985

[6] Ellis, R.; Newman, C.: Limit Theorems for Sums of Dependent Random Variables Occurring in Statistical Mechanics, Z. Wahrsch. Verw. Gebiete 44(2), pp. 117-139 (1978)

[7] Ellis, R.; Newman, C.: The Statistics of Curie-Weiss Models, J. Stat. Phys., $19(2), 149-161(1978)$

[8] Fedele, Micaela: Rescaled Magnetization for Critical Bipartite Mean-Fields Models, J. Stat. Phys. 155:223-226 (2014)

[9] Fedele, Micaela; Contucci, Pierluigi: Scaling Limits for Multi-species Statistical Mechanics Mean-Field Models, J. Stat. Phys. 144:1186-1205 (2011)

[10] Gallo, I.; Barra, A.; Contucci, P.: Scaling Limits for Multi-Species Statistical Mechanics Mean-Field Models, J. Stat. Phys., 144, pp. 1186-1205 (2011) 
[11] Husimi, K.: Statistical Mechanics of Condensation, Proceedings of the International Conference of Theoretical Physics, pp. 531-533, Science Council of Japan, Tokyo (1953)

[12] Isserlis, L.: On a Formula for the Product-Moment Coefficient of any Order of a Normal Frequency Distribution in any Number of Variables, Biometrika 12, pp. 134-139 (1918)

[13] Kac, M.: Mathematical Mechanisms of Phase Transitions, in Statistical Physics: Phase Transitions and Superfluidity, Vol. 1, pp. 241-305, Brandeis University Summer Institute in Theoretical Physics (1968)

[14] Kirsch, Werner: A Survey on the Method of Moments, available from http://www.fernuni-hagen.de/stochastik/

[15] Kirsch, Werner: On Penrose's Square-root Law and Beyond, Homo Oeconomicus 24(3/4): 357-380, 2007

[16] Kirsch, Werner; Toth, Gabor: Two Groups in a Curie-Weiss Model, arxiv.org/abs/1712.08477

[17] Kleiber, Christian; Stoyanov, Jordan: Multivariate distributions and the moment problem. J. Multivariate Anal. 113 (2013), 7-18.

[18] Löwe, Matthias; Schubert, Kristina: Fluctuations for block spin Ising models, Preprint arXiv:1806.06000

[19] Olver, Frank: Asymptotics and special functions, Academic Press (1974)

[20] Temperley, H.N.V.: The Mayer Theory of Condensation Tested against a Simple Model of the Imperfect Gas, Proc. Phys. Soc., A 67, pp. 233-238 (1954)

[21] Thompson, C.J.: Mathematical Statistical Mechanics, Macmillan (1972) 